\title{
High incidence of primary tuberculosis
}

\author{
H J Milburn, J Gibilaro, H Atkinson, R Heathcock
}

\begin{abstract}
An usually high rate of both tuberculosis infection and active disease is reported in 11 of 38 nursery children in contact with a case of smear positive pulmonary tuberculosis, emphasising the susceptibility of young children to this disease. This report also underlines some important principles in case finding and disease control. (Arch Dis Child 2000;82:386-387)
\end{abstract}

Keywords: tuberculosis; incidence; tuberculin conversion; nursery; outbreak

Annual tuberculosis rates in Lambeth are four times the national average at 41 cases per 100000 population. This reflects both the population mix and social problems found in this area of London. Most cases occur in adults of 25-44 years; cases notified in children under 5 years account for under $6 \%$ of total notification (fig 1). In children symptoms of tuberculosis may be few and isolation of organisms difficult. Furthermore, minimal active disease may be unrecognised on chest radiography. ${ }^{1}$ Current notifications in this age group may therefore underestimate actual levels of disease. We report an unusually high rate of both tuberculosis infection and active disease among children in a private London nursery school. This illustrates the susceptibility of this age group to tuberculosis and underlines a number of important principles in disease control.

\section{Subjects, methods, and results}

The nursery was a clean, well run, private establishment. Two rooms were used for different activities but there was no segregation of children and all children mixed with all staff. Most children spent eight hours per day, five days per week in the nursery and had close physical contact with the helpers.

Accepted 6 December 1999

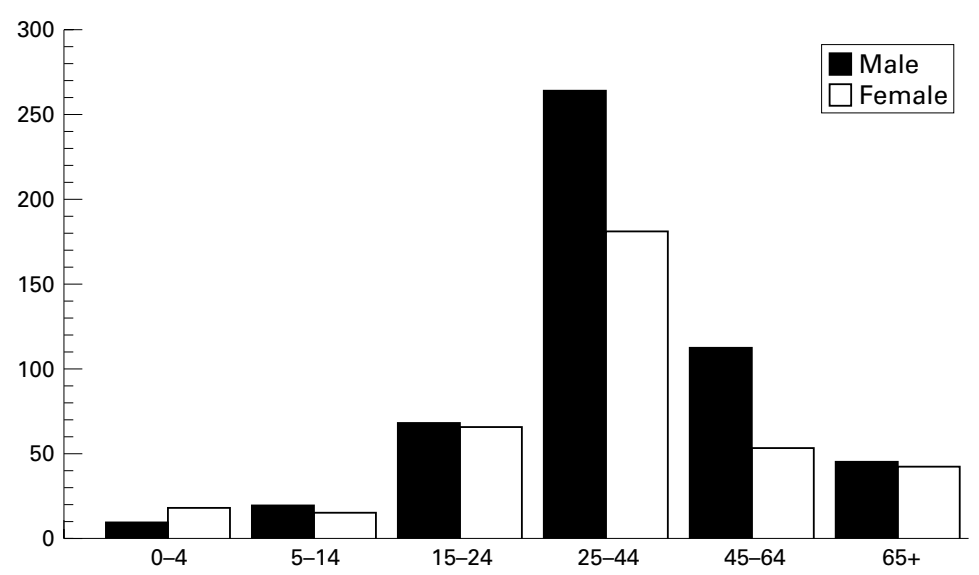

Figure 1 Lambeth, Southwark, and Lewisham enhanced tuberculosis surveillance programme. Tuberculosis notifications by age and sex, 1995-99.
A 25 year old nursery helper presented with a two month history of cough and weight loss. He had entered the UK 14 months earlier from South America. Chest radiograph showed right apical cavitation; sputum was positive $(++)$ for acid fast bacilli on direct smear and grew fully sensitive Mycobacterium tuberculosis on culture.

All 11 staff and 38 children aged $2-4$ years were Heaf tested within two weeks of presentation of the index case and again six weeks later. All staff had had previous BCG vaccinations. Three gave Heaf reactions grade $0-1$ (negative reaction) and eight grade $2-3$ (consistent with previous BCG) with no changes on second testing. All these staff were well and discharged from further follow up. Four children with previous BCG vaccination had appropriate Heaf grades 1 or 2 on both occasions. Eleven children were Heaf grade $0 / 0$ and received BCG. Offer of BCG was refused for 12 further Heaf negative children. Eleven remaining unvaccinated children initially Heaf grade $0-1$, converted their responses to grades $2-4$ on repeat testing. Although none spontaneously complained of symptoms, on questioning five had cough or fever and six had chest radiograph appearances consistent with primary tuberculosis (table 1).

Eight children were treated for tuberculosis with rifampicin, isoniazid, and pyrazinamide for two months followed by rifampicin and isoniazid for four months. Two children with normal chest $x$ ray findings were included. This was because both had a notable increase in Heaf response after six weeks and, as so many other children had obvious signs of disease, it was clear we were dealing with infectious tuberculosis. Seven children gained weight, symptoms resolved, and chest radiographs returned to normal. Even those who denied symptoms initially were reported as being livelier and "much better" on treatment. One child failed to attend for follow up after three months treatment despite repeated visits from the specialist nurse. The final outcome of this child is unknown. Three children received rifampicin and isoniazid chemoprophylaxis for three months. None experienced side effects from the medication. Fifteen close family contacts of the treated children were screened. None had evidence of disease.

\section{Discussion}

All 11 children with tuberculosis infection were diagnosed within two months of presentation of the index case. There was no delay in the index case presenting for treatment or the initiation of screening of staff and children. The risk of infection with tuberculosis depends on the duration and intensity of contact with an infectious case. 
Table 1 Clinical details of 11 children with evidence of tuberculous infection

\begin{tabular}{|c|c|c|c|c|c|c|c|}
\hline Patient & Age (y) & Heaf at $2 w k$ & Heaf at $8 w k$ & $C X R$ & Symptoms, signs & Treatment & Outcome \\
\hline $\mathrm{F}$ & 4 & 1 & 3 & Abnormal & Cough, crackles & Full & Completed, CXR normal, well \\
\hline M & 3 & 1 & 3 & Abnormal & Cough & Full & Completed, CXR normal. well \\
\hline M & 3 & 0 & 2 & Normal & None & Full & Completed, well \\
\hline M & 4 & 0 & 3 & Normal & None & Full & Completed, well \\
\hline $\mathrm{F}$ & 2 & 1 & 2 & Normal & None & Prophylaxis & Completed, well \\
\hline $\mathrm{F}$ & 2 & 1 & 2 & Normal & None & Prophylaxis & Completed, well \\
\hline M & 2 & 0 & 3 & Abnormal & Cough & Full & Completed, well, CXR normal \\
\hline $\mathrm{F}$ & 3 & 0 & 3 & Abnormal & $\begin{array}{l}\text { Cough, fever, anorexia, } \\
\text { nodes }\end{array}$ & Full & Lost to follow up \\
\hline $\mathrm{F}$ & 2.5 & 1 & 4 & Abnormal & Cough, crackles, fever & Full & Completed, well, CXR normal \\
\hline $\mathrm{F}$ & 3 & 1 & +ve Mantoux & Abnormal & None & Full & Completed, well, CXR normal \\
\hline M & 2 & 1 & 2 & Normal & None & Prophylaxis & Completed, well \\
\hline
\end{tabular}

CXR, chest radiograph

Full treatment: rifampicin and isoniazid for six months, plus pyrazinamide for first two months.

Prophylaxis: rifampicin and isoniazid for three months.

Thus, close household contacts are at greatest risk. ${ }^{2}$ Once infected, the chance of developing disease is greatest immediately following infection, declining exponentially thereafter. ${ }^{3}$ Within the first year after infection, the incidence of clinically significant disease is approximately $1.5 \%$ and the cumulative risk of disease during the first five years $5-10 \%$. Among these nursery children, eight of 38 $(21 \%)$ had evidence of primary disease, an extremely high rate. A further three of 38 received prophylaxis for infection. This confirms earlier reports of the greatly increased susceptibility of unvaccinated young children to tuberculosis. ${ }^{45}$

Classical symptoms are uncommon, obtaining appropriate specimens for microbiology is difficult, and overt disease easily missed. We believe, therefore, that disease notification may underestimate actual incidence in children under 5 years. Secondly, contact tracing identifies infected children and others at risk, but relies on prompt notification of the index case as occurred in this incident. Thirdly, the high number (11 of 38) who tuberculin converted confirms the importance of repeat testing in unvaccinated, initially tuberculin negative contacts of smear positive disease. ${ }^{6}$ A number of other important points are raised by this incident. Pre-employment screening for tuberculosis should be a prerequisite for all adults working with vulnerable groups such as the under fives, particularly those originating from areas of high disease prevalence. Most small, private businesses, however, do not have access to occupational health, and it is therefore important to raise public awareness of tuberculosis and to educate those working in such environments as well as health personnel.

Neonatal vaccination with BCG of vulnerable groups is another method of reducing the risks of similar incidents. In Lambeth, neonatal BCG is offered to any child born to parents from a country at high risk, and any child likely to travel to or have visitors from such a country. The majority of infected children in this incident had been born overseas and had missed the opportunity of neonatal BCG. We report these cases as a timely reminder to all who care for young children. Even treatment of "well" children with primary disease reduces future individual morbidity and risk of further spread of infection.

1 Delacourt C, Mani TM, Bonnerot V, de Blic J, Sayeg N, Lallemand D, Scheinmann P. Computed tomography with normal chest radiograph in tuberculous infection. Arch Dis Child 1993;69:430-2.

2 Grzybowski S, Barnett GD, Styblo K. Contacts of cases of active pulmonary tuberculosis. Bull Int Union Tuberc 1975; 50:90-106.

3 Chiba Y, Kurihara T. Development of pulmonary tuberculosis with special reference to the time interval after tuberculin conversion. Bull Int Union Tuberc 1979;54:263-4.

4 Aspin J, Sheldon M. An epidemic of TB in a Staffordshire school. Tubercle 1965;44:321-44.

5 Hill JD, Stephenson DK. TB in un-vaccinated children, adolescents and young adults. BMF 1983;286:1471-3.

6 Joint Tuberculosis Committee of the British Thoracic Society. Control and prevention of tuberculosis in the United Kingdom: Code of practice 1994. Thorax 1994;49:1193200. 